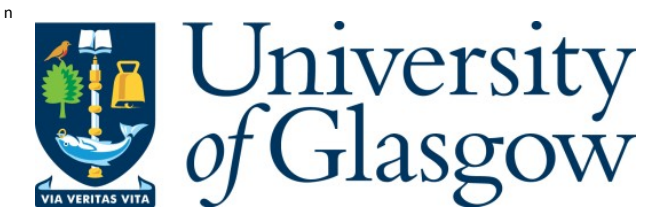

K rysta, P., M anlove, D., R astegari, B ., and Zhang, J. (2014) Size versus truthfulness in the house allocation problem. In: Fifteenth A CM Conference on E conomics and Computation (EC'14), 8-12 J une 2014, Palo A Ito, CA USA.

Copyright @ 2014 A ssociation for Computing M achinery

http://eprints.gla.ac.uk/97926/

Deposited on: 06 October 2014

Enlighten - Research publications by members of the University of Glasgow http://eprints.gla.ac.uk 


\title{
Size versus truthfulness in the House Allocation problem*
}

\author{
Piotr Krysta $^{1}$, David Manlove ${ }^{2}$, Baharak Rastegari ${ }^{2}$ and Jinshan Zhang ${ }^{1}$ \\ ${ }^{1}$ Department of Computer Science, University of Liverpool, \\ Ashton Building, Ashton Street, Liverpool L69 3BX, UK. \\ Email: \{p.krysta, jinshan.zhang\}@liverpool.ac.uk. \\ ${ }^{2}$ School of Computing Science, University of Glasgow, \\ Sir Alwyn Williams Building, Glasgow G12 8QQ, Glasgow, UK. \\ Email: \{david.manlove, baharak.rastegari\}@glasgow.ac.uk.
}

\begin{abstract}
We study the House Allocation problem (also known as the Assignment problem), i.e., the problem of allocating a set of objects among a set of agents, where each agent has ordinal preferences (possibly involving ties) over a subset of the objects. We focus on truthful mechanisms without monetary transfers for finding large Pareto optimal matchings. It is straightforward to show that no deterministic truthful mechanism can approximate a maximum cardinality Pareto optimal matching with ratio better than 2. We thus consider randomized mechanisms. We give a natural and explicit extension of the classical Random Serial Dictatorship Mechanism (RSDM) specifically for the House Allocation problem where preference lists can include ties. We thus obtain a universally truthful randomized mechanism for finding a Pareto optimal matching and show that it achieves an approximation ratio of $\frac{e}{e-1}$. The same bound holds even when agents have priorities (weights) and our goal is to find a maximum weight (as opposed to maximum cardinality) Pareto optimal matching. On the other hand we give a lower bound of $\frac{18}{13}$ on the approximation ratio of any universally truthful Pareto optimal mechanism in settings with strict preferences. In the case that the mechanism must additionally be non-bossy, an improved lower bound of $\frac{e}{e-1}$ holds. This lower bound is tight given that RSDM for strict preference lists is non-bossy. We moreover interpret our problem in terms of the classical secretary problem and prove that our mechanism provides the best randomized strategy of the administrator who interviews the applicants.
\end{abstract}

Keywords: House allocation problem; Assignment problem; Pareto optimal matching; Randomized mechanisms; Truthfulness

\section{Introduction}

We study the problem of allocating a set of indivisible objects among a set of agents. Each agent has private ordinal preferences over a subset of objects - those they find acceptable, and each agent may be allocated at most one object. This problem has been studied by both economists and computer scientists. When monetary transfers are not permitted,

\footnotetext{
*Supported by grants EP/K01000X/1 and EP/K010042/1 from the Engineering and Physical Sciences Research Council.
} 
the problem is referred to as the House Allocation problem (henceforth abbreviated by HA; see, e.g., $[19,1,32]$ ) or the Assignment problem (see, e.g., $[18,9])$ in the literature. In this paper we opt for the term House Allocation problem. Most of the work in the literature assumes that the agents have strict preferences over their acceptable objects. It often happens though that an agent is indifferent between two or more objects. Here we let agents express indifference, and hence preferences may involve ties unless explicitly stated otherwise.

It is often desired that as many objects as possible become allocated among the agents - i.e., an allocation of maximum size is picked, hence making as many agents happy as possible. Usually, depending on the application of the problem, we are required to consider some other optimality criteria, sometimes instead of and sometimes in addition to maximizing the size of the allocation. Several optimality criteria have been considered in the HA setting, and perhaps the most studied such concept is Pareto optimality (see, e.g., $[1,2,14,12,28])$, sometimes referred to as Pareto efficiency. Economists, in particular, regard Pareto optimality as the most fundamental requirement for any "reasonable" solution to a non-cooperative game. Roughly speaking, an allocation $\mu$ is Pareto optimal if there does not exist another allocation $\mu^{\prime}$ in which no agent is worse off, and at least one agent is better off, in $\mu^{\prime}$. In this work we are mainly concerned with Pareto optimal allocations of maximum size, but will also consider weighted generalisations.

The related Housing Market problem (HM) is the variant of HA in which there is an initial endowment, i.e., each agent owns a unique object initially (in this case the numbers of agents and objects are usually defined to be equal). In this setting, the most widely studied solution concept is that of the core, which is an allocation of agents to objects satisfying the property that no coalition $C$ of agents can improve (i.e., every agent in $C$ is better off) by exchanging their own resources (i.e., the objects they brought to the market). In the case of strict preferences, the core is always non-empty [26], unique, and indeed Pareto optimal. When preferences may include ties, the notion of core that we defined is sometimes referred to as the weak core. In this case a core allocation need not be Pareto optimal, but Saban and Sethuraman [29] gave an efficient algorithm for finding a core allocation that does additionally satisfy Pareto optimality. Our problem differs from HM in that there is no initial endowment, and hence our focus is on Pareto optimal matchings rather than outcomes in the core.

For strictly ordered lists, Abraham et al. [2] gave a characterisation of Pareto optimal matchings that led to an $O(m)$ algorithm for checking an arbitrary matching for Pareto optimality, where $m$ is the total length of the agents' preference lists. This characterisation was extended to the case that preference lists may include ties by Manlove [22, Sec. 6.2.2.1], also leading to an $O(m)$ algorithm for checking a matching for Pareto optimality. For strictly ordered preference lists, a maximum cardinality Pareto optimal matching can be found in $O\left(\sqrt{n_{1}} m\right)$ time, where $n_{1}$ is the number of agents [2]. The fastest algorithm currently known for this problem when preference lists may include ties is based on minimum cost maximum cardinality matching and has complexity $O(\sqrt{n} m \log n)$ (see, e.g., [22, Sec. 6.2.2.1], where $n$ is the total number of agents and objects.

As stated earlier, agents' preferences are private knowledge. Hence, unless they find it in their own best interests, agents may not reveal their preferences truthfully. An allocation mechanism is truthful if it gives agents no incentive to misrepresent their preferences. Perhaps unsurprisingly, a mechanism based on constructing a maximum cardinality Pareto optimal allocation is manipulable by agents misrepresenting their preferences (Theorem 2.1 in Section 2). Hence, we need to make a compromise and weaken at least one of these requirements. In this work, we relax our quest for finding a maximum cardinality Pareto optimal allocation by trading off the size of a Pareto optimal allocation against truthful- 
ness; more specifically, we seek truthful mechanisms that provide good approximations to the maximum size.

Under strict preferences, Pareto optimal matchings can be computed by a classical algorithm called the Serial Dictatorship Mechanism (SDM) - see, e.g., [1], also referred to as the Priority Mechanism - see, e.g., [9]. SDM is a straightforward greedy algorithm that takes each agent in turn and allocates him to the most preferred available object on his preference list. Precisely due to this greedy approach, SDM is truthful. Furthermore, SDM is guaranteed to find a Pareto optimal allocation at least half the size of a maximum one, merely because any Pareto optimal allocation is at least half the size of a maximum one (see, e.g., [2]). Hence, at least in the case of strict preferences, we are guaranteed an approximation ratio of 2. Can we do better? It turns out that if we stay in the realm of deterministic mechanisms, a 2-approximation is the best we can hope for (Theorem 2.1, Section 2).

Hence we turn to randomized mechanisms in order to achieve a better approximation ratio. The obvious candidate to consider is the Random Serial Dictatorship Mechanism (RSDM) (see, e.g., [1]), also known as the Random Priority mechanism (see, e.g., [9]), that is defined for settings with strict preferences. RSDM randomly generates an ordering of the agents and then proceeds by running SDM relative to this ordering. It is straightforward to extend results from [8] in order to show that RSDM has an approximation ratio of $\frac{e}{e-1}$ (relative to the size of a maximum cardinality Pareto optimal matching).

When indifference is allowed, finding a Pareto optimal allocation is not as straightforward as for strict preferences. For example, one may consider breaking the ties randomly and then applying SDM. This approach, unfortunately, may produce an allocation that is not Pareto optimal (see beginning of Section 3). Few works in the literature considered extensions of SDM to the case where agents' preferences may include ties. However Bogomolnaia and Moulin [10] and Svensson [30] provide an implicit extension of SDM (in the former case for dichotomous preferences ${ }^{1}$ ) but do not describe how one may actually implement the algorithm. Aziz et al. [4] provide an explicit extension for a more general class of problems, including HA. Pareto optimal matchings in HA can also be found by reducing to the setting with initial endowments [20] by defining dummy objects as endowments for the agents. This gives a truthful Pareto optimal mechanism for our problem with run time $O\left(n_{1}^{2} \gamma+n_{1}^{2} \log n_{1}\right)[29]$.

Contributions of this paper. In this paper we provide a natural and explicit extension of SDM for the setting in which preferences may exhibit indifference. We argue that our extension is more intuitive than that of Aziz et al. [4] when considering specifically HA. Moreover, as the mechanism of Saban and Sethuraman [29] does not consider the agents sequentially, it is difficult to analyse its approximation guarantee. Our algorithm runs in time $O\left(n_{1}^{2} \gamma\right)$, where $\gamma$ is the maximum length of a tie in any agent's preference list. This is faster than the algorithm in [29] when $\gamma<n_{1}$. We prove the following results that involve upper and lower bounds for the approximation ratio (relative to the size of a maximum cardinality Pareto optimal matching) of randomized truthful mechanisms for computing a Pareto optimal matching:

1. By extending RSDM to the case of preference lists with ties, we give a universally truthful randomized mechanism ${ }^{2}$ for finding a Pareto optimal matching that has an approximation ratio of $\frac{e}{e-1}$ with respect to the size of a maximum cardinality Pareto optimal matching.

\footnotetext{
${ }^{1}$ An agent's preference list is dichotomous if it comprises a single tie containing all acceptable objects.

${ }^{2} \mathrm{~A}$ randomized mechanism is universally truthful if it is a probability distribution over truthful deterministic mechanisms. This is the strongest known notion of truthfulness for randomized mechanisms.
} 
2. We give a lower bound of $\frac{18}{13}$ on the approximation ratio of any universally truthful Pareto optimal mechanism in settings with strict preferences. If the mechanism must additionally be non-bossy ${ }^{3}$, we observe that [7] implies an improved lower bound of $\frac{e}{e-1}$. This lower bound is tight given that the classical RSDM mechanism for strict preferences is non-bossy.

3. We extend RSDM to the setting where agents have priorities (weights) and our goal is to find a maximum weight (as opposed to maximum cardinality) Pareto optimal matching. Our mechanism is universally truthful and guarantees a $\frac{e}{e-1}$ approximation with respect to the weight of a maximum weight Pareto optimal matching.

4. We finally observe that our problem has an "online" or sequential flavour similar to secretary problems ${ }^{4}$. Given this interpretation, we prove that our mechanism uses the best random strategy of interviewing the applicants in the sense that any other strategy would lead to an approximation ratio worse than $\frac{e}{e-1}$ (see also below under related work).

Discussion of technical contributions. Indifferences in agents' preference lists introduce major technical difficulties. When extending SDM from strict preferences to preferences with ties, we first present an intuitive mechanism, called SDMT-1, based on the idea of augmenting paths. It is relatively easy to prove that SDMT-1 is Pareto optimal and truthful. However, it is difficult to analyse the approximation guarantee of the randomized version of SDMT-1. For this purpose we build on the primal-dual analysis of Devanur et al. [15]. They employ a linear programming (LP) relaxation of the bipartite weighted matching problem. They prove that their dual solution is feasible in expectation for the dual LP and use it to show the approximation guarantee. Towards this goal they prove two technical lemmas, a dominance lemma and monotonicity lemma. The randomized version of SDMT-1 uses random variables $Y_{i}$ for each agent $i \in N$ to generate a random order in which agents are considered. Considering agent $i$ and fixed values of the random variables $Y_{-i}$ of all other agents, Devanur et al. [15] define a threshold $y^{c}$ which as $Y_{i}$ varies determines when agent $i$ is matched (to an object) or unmatched (dominance lemma). The monotonicity lemma shows how values of the dual LP variables change when $Y_{i}$ varies. To extend the definition of $y^{c}$, we need to remember the structure of all potential augmenting paths in SDMT-1, and for this purpose we introduce a second mechanism, SDMT-2. Interestingly, SDMT-2 is inspired by the idea of top trading cycle mechanisms, see, e.g., [29], however it retains the "sequential" nature of SDMT-1. The two mechanisms, SDMT-1 and SDMT-2, are equivalent: they match the same agents, giving them objects from the same ties. This implies that SDMT-2 is also truthful and Pareto optimal. SDMT-2 is the key to defining the threshold $y^{c}$ : its running time is no worse than that of SDMT-1, but it implicitly maintains all relevant augmenting paths arising from agents' ties. We prove the monotonicity and dominance lemmas for SDMT-2 by carefully analysing the structure of frozen subgraphs that are generated from the relevant augmenting paths; here frozen

\footnotetext{
${ }^{3} \mathrm{~A}$ deterministic mechanism in settings with strict preferences is non-bossy if no agent can misreport his preferences in such a way that his allocation is not changed but the allocation of some other agent is changed.

${ }^{4}$ In the secretary problem, an administrator is willing to hire the best secretary out of $n$ rankable applicants for a position. The applicants are interviewed one-by-one in random order. A decision about each particular applicant is to be made immediately after the interview. Once rejected, an applicant cannot be recalled. During the interview, the administrator can rank the applicant among all applicants interviewed so far, but is unaware of the quality of yet unseen applicants. The question is about the optimal strategy to maximize the probability of selecting the best applicant.
} 
roughly means that they will not change subsequently. This new approach may find further applications for primal-dual analysis of mechanisms for assignment problems where agents have indifferences. Finally, we would like to highlight that our proof of an $\frac{18}{13}$ lower bound on the approximation ratio of any universally truthful and Pareto optimal mechanism uses Yao's minmax principle and an interesting case analysis to account for all such possible mechanisms.

Related work. This work can be placed in the context of designing truthful approximate mechanisms for problems in the absence of monetary transfer [24]. Bogomolnaia and Moulin [9] designed a randomized weakly truthful and envy-free mechanism, called the Probabilistic Serial mechanism (PS), for HA with complete lists. Very recently the same authors considered the same approximation problem as ours but in the context of envy-free rather than truthful mechanisms, and for strict preference lists and unweighted agents [11]. They showed that PS has an approximation ratio of $\frac{e}{e-1}$, which is tight for any envyfree mechanism. Bhalgat et al. [8] investigated the social welfare of PS and RSDM. Tight deterministic truthful mechanisms for weighted matching markets were proposed by Dughmi and Ghosh [17] and they also presented an $O(\log n)$-approximate random truthful mechanism for the Generalized Assignment Problem (GAP) by reducing, with logarithmic loss in the approximation, to the solution for the value-invariant GAP. Aziz et al. [5] studied notions of fairness involving the stochastic dominance relation in the context of HA, and presented various complexity results for problems involving checking whether a fair assignment exists. Chakrabarty and Swamy [13] proposed rank approximation as a measure of the quality of an outcome and introduced the concept of lex-truthfulness as a notion of truthfulness for randomized mechanisms in HA.

The weighted version of our problem is related to two widely studied online settings, known in the literature as the online vertex-weighted bipartite matching problem [3] and secretary problems [6]. In our problem the administrator holds all the objects (they can be thought of as available positions), and all agents with unknown preference lists are applicants for these objects. Each applicant also has a private weight, which can be thought of as their quality (reflecting the fact that some of an agent's skills may not be evident from their $\mathrm{CV}$, for example). However we assume that they cannot overstate their weights (skills), because they might be checked and punished. This is similar to the classical assumption of no overbidding (e.g., in sponsored search auctions). Applicants are interviewed one-by-one in a random order. When an applicant arrives he chooses his most-preferred available object and the decision as to whether it is allocated to him is made immediately, and cannot be changed in the future.

Our weighted agents correspond to weighted vertices in the vertex-weighted bipartite matching context, but our objects do not arrive online as in the setting of [3]. However, if the preference ordering of each agent in our setting, over his acceptable objects, coincides with the arrival order of the objects in [3], then the two problems are the same. In the transversal matroid secretary problem, see, e.g., [16], objects are known in advance as in our setting, weighted agents arrive in a (uniform) random order, and the goal is to match them to previously unmatched objects. The administrator's goal is the optimal strategy, which is the random arrival order of agents, to maximize the ratio between the total weight of matched agents and the maximum weight of a matching if all the applicants' preference lists are known in advance. We show that even if the weights of all agents are the same, our algorithm uses the best possible random strategy; no other such strategy leads to better than $\frac{e}{e-1}$-approximate matching. 
Organization of the paper. The remainder of the paper is organized as follows. In Section 2 we define notation and terminology used in this paper, and show the straightforward lower bound for the approximation ratio of deterministic truthful mechanisms. SDMT-1 and SDMT-2 are presented in Sections 3 and 4 respectively, and in the latter section it is proved that the two mechanisms are essentially equivalent. The approximation ratio of $\frac{e}{e-1}$ for the randomized version of the two mechanisms is established in Section 5, whilst Section 6 contains our lower bound results. Finally, some concluding remarks are given in Section 7. All missing proofs can be found in the full version of this paper [21].

\section{Definitions and preliminary observations}

Let $N=\left\{1,2, \cdots, n_{1}\right\}$ be a set of $n_{1}$ agents and $A$ be a set of $n_{2}$ objects. Let $n=n_{1}+n_{2}$. Let $[i]$ denote the set $\{1,2, \cdots, i\}$. We assume that each agent $i \in N$ finds a subset of objects acceptable and has a preference ordering, not necessarily strict, over these objects. We write $a_{t} \succ_{i} a_{s}$ to denote that agent $i$ strictly prefers object $a_{t}$ to object $a_{s}$, and write $a_{t} \simeq_{i} a_{s}$ to denote that $i$ is indifferent between $a_{t}$ and $a_{s}$. We use $a_{t} \succeq_{i} a_{s}$ to denote that agent $i$ either strictly prefers $a_{t}$ to $a_{s}$ or is indifferent between them, and say that $i$ weakly prefers $a_{t}$ to $a_{s}$. In some cases a weight $w_{i}$ is associated with each agent $i$, representing the priority or importance of the agent. Weights need not be distinct. Let $W=\left(w_{1}, w_{2}, \ldots, w_{n_{1}}\right)$.

We assume that the indifference relation is transitive. This implies that each agent essentially divides his acceptable objects into different bins or indifference classes such that he is indifferent between the objects in the same indifference class and has a strict preference ordering over these indifference classes. For each agent $i$, let $C_{k}^{i}, 1 \leq k \leq n_{2}$, denote the $k$ th indifference class, or tie, of agent $i$. We also assume that if there exists $l \in\left[n_{2}\right]$, where $C_{l}^{i}=\emptyset$, then $C_{q}^{i}=\emptyset, \forall q, l \leq q \leq n_{2}$. We let $L(i)=\left(C_{1}^{i} \succ_{i} C_{2}^{i} \succ_{i} \cdots \succ_{i}\right.$ $C_{n_{2}}^{i}$ ) and call $L(i)$ the preference list of agent $i$. We abuse notation and write $a \in L(i)$ if $a$ appears in preference list $L(i)$, i.e., if agent $i$ finds object $a$ acceptable. We say that $i$ ranks object $a k$ th if $a \in C_{k}^{i}$. We denote by $\operatorname{rank}(i, a)$ the rank of object $a$ in agent $i$ 's preference list and let $\operatorname{rank}(i, a)=n_{2}+1$ if $a$ is not acceptable to $i$. Therefore $a_{t} \succ_{i} a_{s}$ if and only if $\operatorname{rank}\left(i, a_{t}\right)<\operatorname{rank}\left(i, a_{s}\right)$, and $a_{t} \simeq_{i} a_{s}$ if and only if $\operatorname{rank}\left(i, a_{t}\right)=\operatorname{rank}\left(i, a_{s}\right)$.

Let $L=\left(L(1), L(2), \cdots, L\left(n_{1}\right)\right)$ denote the joint preference list profile of all agents. We write $L(-i)$ to denote the joint preference list profile of all agents except agent $i$; i.e. $L(-i)=\left(L(1), \ldots, L(i-1), L(i+1), \ldots, L\left(n_{1}\right)\right)$. Let $\mathcal{L}$ denote the set of all possible joint preference list profiles. An instance of HA is denoted by $I=(N, A, L, W)$. We drop $W$ and write $I=(N, A, L)$ if we are dealing with an instance where agents are not assigned weights, or equivalently if they all have the same weight. Let $\mathcal{I}$ denote the set of all possible instances of HA.

A matching $\mu$ is a subset of $N \times A$ such that each agent and object appears in at most one pair of $\mu$. If $(i, a) \in \mu$, agent $i$ and object $a$ are said to be matched together. If $(i, a) \in \mu$ for some $a$, we say that $i$ is matched, and unmatched otherwise. The definitions of matched and unmatched for an object are analogous. If agent $i$ is matched, $\mu(i)$ denotes the object matched to $i$. Similarly if object $a$ is matched, $\mu^{-1}(a)$ denotes the agent matched to $a$. In what follows, we will consider the following undirected graph $G=(V, E)$ where $V=(N \cup A)$ and $E=\{(i, a), i \in N, a \in L(i)\}$. We also use $\mu$ to denote a matching (in the standard graph-theoretic sense) in $G$. The size of a matching $\mu$ is equal to the number of agents matched under $\mu$. In the presence of weights, the weight of a matching is equal to the sum of the weights of the matched agents.

For two given matchings $\mu_{1}, \mu_{2}$, we will frequently use $\mu_{1} \oplus \mu_{2}$ to denote the symmetric difference with respect to their sets of edges. An alternating path in $G$, given a matching 
$\mu_{1}$, is a path that consists of edges that alternately belong to $\mu_{1}$ and do not belong to $\mu_{1}$. An augmenting path is an alternating path where the first and the last vertices on the path are unmatched in $\mu_{1}$. To augment along an augmenting path, given matching $\mu_{1}$, means that a new matching $\mu_{2}$ is created by removing edges on the path that belong to $\mu_{1}$ and adding edges on the path that do not belong to $\mu_{1}$.

A matching $\mu$ is Pareto optimal if there is no other matching under which some agent is better off while none is worse off. Formally, $\mu$ is Pareto optimal if there is no other matching $\mu^{\prime}$ such that (i) $\mu^{\prime}(i) \succeq_{i} \mu(i)$ for all $i \in N$, and (ii) $\mu^{\prime}\left(i^{\prime}\right) \succ_{i^{\prime}} \mu\left(i^{\prime}\right)$ for some agent $i^{\prime} \in N$.

Let $\mathcal{M}$ denote the set of all possible matchings. A deterministic mechanism $\phi$ maps an instance of HA to a matching, i.e. $\phi: \mathcal{I} \rightarrow \mathcal{M}$. Let $R: \mathcal{M} \rightarrow[0,1]$ denote a distribution over possible matchings (which we also call a random matching); i.e. $\sum_{\mu \in \mathcal{M}} R(\mu)=1$. A randomized mechanism $\phi$ is a mapping from $\mathcal{I}$ to a distribution over possible matchings, i.e., $\phi: \mathcal{I} \rightarrow \operatorname{Rand}(\mathcal{M})$, where $\operatorname{Rand}(\mathcal{M})$ is the set of all random matchings. A deterministic mechanism is Pareto optimal if it returns a Pareto optimal matching. A randomized mechanism is Pareto optimal if it returns a distribution over Pareto optimal matchings.

Agents' preferences are private knowledge and an agent may prefer not to reveal his preferences truthfully if it is not in his best interests, for a given mechanism. A deterministic mechanism is dominant strategy truthful (or truthful) if agents always find it in their best interests to declare their preferences truthfully, no matter what other agents declare, i.e., for every agent $i$ and every possible declared preference list $L^{\prime}(i)$ for $i, \phi(L(i), L(-i)) \succeq_{i} \phi\left(L^{\prime}(i), L(-i)\right), \forall L(i), \forall L(-i)$. A randomized mechanism $\phi$ is universally truthful if it is a probability distribution over deterministic truthful mechanisms.

Denote by $w(\phi(I))$ the (expected) weight of the (random) matching generated by mechanism $\phi$ on instance $I \in \mathcal{I}$, and by $w(I)$ the weight of a maximum weight Pareto optimal matching in $I$. The approximation ratio of $\phi$ is then defined as $\max _{I \in \mathcal{I}} \frac{w(I)}{w(\phi(I))}$. We give a straightforward lower bound for the approximation ratio of any deterministic truthful mechanism for HA with strict preferences.

Theorem 2.1. No deterministic truthful Pareto optimal mechanism for HA can achieve approximation ratio better than 2. The result holds even for strict preferences.

Proof. Consider a setting with two agents, 1 and 2, and two objects, $a_{1}$ and $a_{2}$. Assume that both agents have weight 1 and prefer $a_{1}$ to $a_{2}$. This setting admits two Pareto optimal matchings, both of size (weight) 2. Assume, for a contradiction, that there exists a truthful Pareto optimal mechanism $\phi$ with approximation ratio strictly smaller than 2 . Hence, in the given setting, $\phi$ must pick one of the two matchings of size 2. Assume, without loss of generality, that $\phi$ picks $\mu=\left\{\left(1, a_{2}\right),\left(2, a_{1}\right)\right\}$. Now, assume that 1 misrepresents his acceptable objects and declares $a_{1}$ as the only object acceptable to him. As $\phi$ is truthful, it must not assign $a_{1}$ to 1 , or else 1 finds it in his best interests to misrepresent his preferences as he prefers his allocated object under the new setting to his allocated object under the original setting. Hence $\phi$ must return a matching of size at most 1 (by assigning an object to 2). However, the new setting admits a Pareto optimal matching of size 2, $\mu^{\prime}=\left\{\left(1, a_{1}\right),\left(2, a_{2}\right)\right\}$. Therefore the approximation ratio of $\phi$ is 2 , a contradiction.

As mentioned in Section 1, the upper bound of 2 is achievable via SDM for HA with strict preferences. If weights and ties exist, simply ordering the agents in decreasing order of their weights and running SDMT-1 (see Algorithm 1 in Section 3) or SDMT-2 (see Algorithm 2 in Section 4) gives a deterministic truthful and Pareto optimal mechanism with approximation ratio 2 . This resolves the problem for deterministic mechanisms and motivates looking into relaxing our requirements. In the following sections we look for randomized truthful mechanisms that construct 'large' weight Pareto optimal matchings. 


\section{First truthful mechanism: SDMT-1}

When preferences are strict, SDM produces a Pareto optimal matching. It turns out that when indifference is allowed, finding an arbitrary Pareto optimal matching is not as straightforward as in the case of strict preferences. Take the following straightforward extension of SDM: given the agents' preference lists, break the ties arbitrarily and then feed the resulting strict preferences to SDM. This mechanism unfortunately may fail to produce a Pareto optimal matching. To see this, consider a setting with two agents, 1 and 2 , and two objects, $a_{1}$ and $a_{2}$. Assume that 1 finds both objects acceptable and is indifferent between them, and that 2 finds only $a_{1}$ acceptable. The only Pareto optimal matching for this setting is $\mu=\left\{\left(1, a_{2}\right),\left(2, a_{1}\right)\right\}$. Assume that 1 is served first and that, after the tie-breaking stage, 1 prefers $a_{1}$ to $a_{2}$. SDM then assigns $a_{1}$ to 1 and leaves 2 unmatched, producing a matching that is not Pareto optimal.

In this section we introduce SDMT-1, Serial Dictatorship Mechanism with Ties, a mechanism that generalizes SDM to the case where agents' preferences may involve ties. SDMT-1 is truthful and is guaranteed to produce a Pareto optimal matching.

Let $I=(N, A, L)$ be an instance of $\mathrm{HA}$, and let a fixed order $\sigma$ of the agents be given. Assume, w.l.o.g., that $\sigma(i)=i$ for all agents $i \in N$. The formal description of SDMT-1 is given in Algorithm 1; an informal description follows. SDMT-1 constructs an undirected bipartite graph $G=(V, E)$ where $V=N \cup A$ and the set of edges $E$ changes during the execution of SDMT-1. The mechanism proceeds in $n_{1}$ phases and returns a matching $\mu$. Each phase of the algorithm corresponds to one iteration of the for loop in Algorithm 1. Let $\mu_{i}$ denote the matching at the end of phase $i$. Initially $E=\emptyset$ and $\mu=\emptyset$. In each phase $i$, agent $i$ is considered and the objects in agent $i$ 's preference list are examined in the order of the indifference classes they belong to. When objects $a \in C_{\ell}^{i}$ are examined, edges $(i, a)$ are provisionally added to $E$ for all $a \in C_{\ell}^{i}$. We then check whether there is an augmenting path in $G$ that starts from agent $i$. If such an augmenting path exists, we augment along that path and modify $\mu$ accordingly. This would mean that agent $i$ is assigned $a$ and every other agent $j$ matched under $\mu_{i-1}$ is assigned an object that he ranks in the same indifference class as $\mu_{i-1}(j)$. Otherwise - if $G$ admits no augmenting path, edges $(i, a)$ are removed from $E$ for all $a \in C_{\ell}^{i}$.

Notice that, at any stage of the mechanism, an edge $(i, a)$ belongs to $E$ if and only if either agent $i$ is matched in $\mu$ and $a \simeq_{i} \mu(i)$ or SDMT- 1 is at phase $i$ and examining the indifference class to which $a$ belongs. Therefore, it is fairly straightforward to observe the following.

Observation 3.1. At the end of phase $i$ of SDMT-1, if agent $i$ is assigned no object then he will be assigned no object when SDMT-1 terminates. Otherwise, if $i$ is provisionally assigned an object a, then he will be allocated an object that he ranks the same as a in the final matching.

Before proceeding to prove our main claim, that SDMT-1 is truthful and produces a Pareto optimal matching, let us discuss a relevant concept that is both interesting in its own right and useful in the proofs that follows. In practice agents may have priorities and the mechanism designer may wish to ensure that the agents with higher priorities are served before satisfying those with lower priorities. Roth et al. [27] studied this concept under the term priority matchings in the case where each agent's preference list is one big tie. This work was motivated by the kidney exchange problem in which patients are assigned priorities based on various criteria; e.g. children and hard-to-match patients have higher priorities. Prior to Roth et al. [27], Svensson [30] studied a similar concept under the name queue allocation in a setting similar to ours. We formally define this concept 


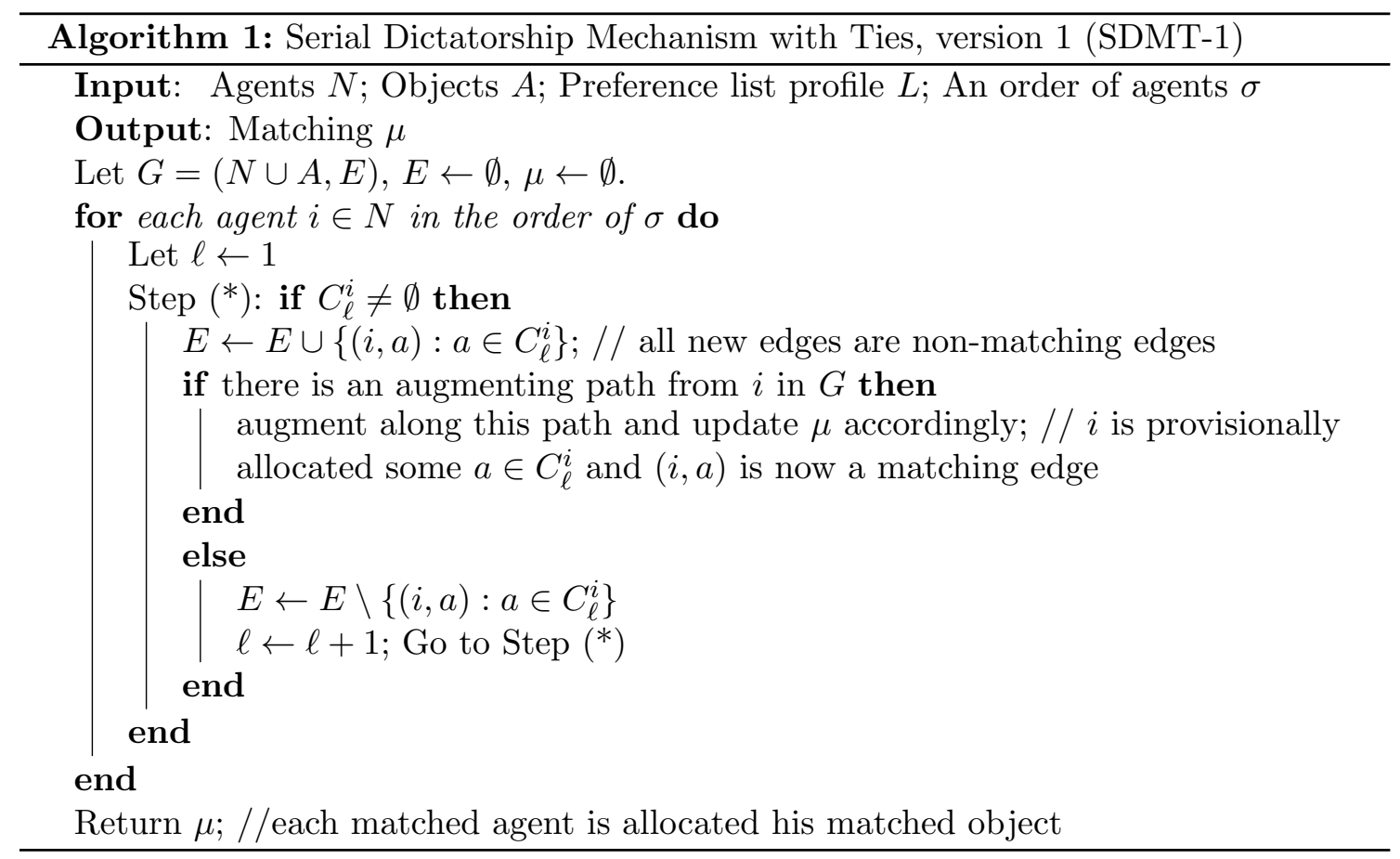

using the terminology strong priority matching, reflecting both the definition in [27] and the fact that preference lists are more general than single ties.

Fix an ordering of the agents $\sigma=i_{1}, \ldots, i_{n_{1}}$. For each matching $\mu$, the signature of $\mu$, denoted by $\rho(\mu)$, is a vector $\left\langle\rho_{1}, \ldots, \rho_{n_{1}}\right\rangle$ where for each $k \in\left[n_{1}\right], \rho_{k}=\operatorname{rank}\left(i_{k}, \mu\left(i_{k}\right)\right)$ if $i_{k}$ is matched under $\mu$, and $\rho_{k}=n_{2}+1$ otherwise. A matching $\mu$ is a strong priority matching (SPM) w.r.t. $\sigma$ if $\mu$ has the lexicographically smallest signature. It is easy to see that a given setting may admit more than one SPM w.r.t. $\sigma$, but all of them have the same signature. When $\sigma$ is fixed and known, we simply say that $\mu$ is a strong priority matching.

Theorem 3.2. The matching produced by SDMT-1 is a strong priority matching w.r.t. $\sigma$.

Proof. Assume, for a contradiction, that the claim does not hold. Hence $\mu$ is not a SPM in $I$. Let $\mu^{*}$ be a SPM in $I$. Let $z$ be the lowest indexed agent (and therefore first in $\sigma$ ) who prefers his partner under $\mu^{*}$ to his partner under $\mu$, i.e. $\mu^{*}(z) \succ_{z} \mu(z)$ and $\mu^{*}(x) \simeq_{x} \mu(x)$, $\forall x<z$ (we denote this deduction by D1). Therefore, in phase $z$ of SDMT-1 no augmenting path has been found starting from $(z, a)$ where $a \succeq_{z} \mu^{*}(z)$ (we denote this deduction by D2). Also, it follows from D1 and Observation 3.1 that, $\mu^{*}(x) \simeq_{x} \mu_{z-1}(x), \forall x<z$ (we denote this deduction by D3). Let $G^{*}$ denote the graph $G$ in phase $z$ during the examination of the indifference class to which $\mu^{*}(z)$ belongs. By D2, $G^{*}$ must admit no augmenting path w.r.t. $\mu_{z-1}$. We show, however, that $G^{*}$ admits an augmenting path starting from $z$. To see this note that, by $\mathbf{D} 1$ and the construction of edges $E$, edges $\left(x, \mu^{*}(x)\right)$ belong to $G^{*}$. If $\mu^{*}(z)$ is unmatched in $\mu_{z-1}$ then $\left(z, \mu^{*}(z)\right)$ constitutes an augmenting path of size 1 in $G^{*}$. Otherwise, let $i_{1}$ denote the partner of $\mu^{*}(z)$ under $\mu_{z-1}$ (note that $i_{1}<z$ ). It follows, from D3 and the construction of $E$, that $i_{1}$ is matched under $\mu^{*}$. If $\mu^{*}\left(i_{1}\right)$ is unmatched under $\mu_{z-1}$ then we have found an augmenting path of length 3. Otherwise, we continue with this argument. As there are a finite number of agents and objects, we have to reach an object $a$ who is unmatched under $\mu_{z-1}$, hence exposing an augmenting path in $G^{*}$, a contradiction. 
Corollary 3.3. The matching produced by SDMT-1 is a Pareto optimal matching.

Proof. By Theorem 3.2, SDMT-1 produces a strong priority matching. It follows from Theorems 1 and 2 in [30] that any strong priority matching is a Pareto optimal matching. Hence, the matching produced by SDMT-1 is a Pareto optimal matching.

SDMT-1 is truthful, no matter which augmenting path is selected in each phase of the mechanism, as the next result shows.

Theorem 3.4. The mechanism SDMT-1 is truthful, and it terminates in time $O\left(n_{1}^{2} \gamma\right)$.

\section{Randomized mechanism with weights and ties}

\subsection{Online interpretation of our problem}

In this section we will analyse our mechanism for the weighted version of our problem. We can interpret it as having an "online" flavour in the following way. An administrator holds all the objects, and all agents with unknown preference lists are applicants for these objects. We assume that weights are private information of each agent, but that they cannot overstate their weights. Applicants are interviewed one-by-one in a random order. A decision about each particular applicant is to be made immediately after the interview. During the interview, the applicant selects his favorite (because we only consider truthful mechanisms) object among the available remaining objects if there exists one in his preference list and is allocated (matched to) that object. ${ }^{5}$ This applicant will not be interviewed again. The administrator can know the number of matched applicants interviewed so far, but is unaware whether yet unseen applicants will be matched or not. Our goal is to find the optimal strategy, which is the random arrival order of agents, that maximizes the ratio between the total weight of matched agents and the maximum weight Pareto optimal matching if all the applicants' preference lists are known in advance.

Our algorithm in the next section is truthful with respect to agents' preferences and weights (under no-overbidding assumption) and provides an $\frac{e}{e-1}$-approximate Pareto optimal matching. We will show in Section 6 that, even if the weights of all agents are the same our algorithm uses the best possible random strategy - no other such strategy leads to better than $\frac{e}{e-1}$-approximate matching.

\subsection{Second truthful mechanism: SDMT-2}

The approximation ratio analysis of the randomized version of SDMT-1 is complex, because it requires additional information which is not maintained by SDMT-1. For the sake of the analysis, we introduce a variant of SDMT-1, called SDMT-2. After introducing some terminology we present SDMT-2, and then establish the equivalence between SDMT-1 and SDMT-2. Pareto optimality and truthfulness of SDMT-2 will then follow from this equivalence and these same two properties of SDMT-1. We will prove that the randomized version of SDMT-2 is $\frac{e}{e-1}$-approximate. By the equivalence of the two algorithms, a randomized version of SDMT-1 has the same approximation ratio.

Let $a_{1} \succ a_{2} \succ \cdots \succ a_{n_{2}}$ be a common order of all the objects; it will be used to break possible ties in SDMT-2). In the course of our algorithm agents will be (temporarily)

\footnotetext{
${ }^{5}$ We can extend this setting to the case where the administrator can decide whether to let the applicant select his favorite object or to reject this applicant, meaning that the applicant gets nothing. In this more general problem, it is not difficult to prove that for any fixed order of the applicants, the decision that the administrator does not reject any applicant will maximize the number of matched applicants. Therefore, this more general problem is reduced to the setting where the administrator lets each applicant select his favorite object, and therefore our lower bound from Section 6 also applies to this setting.
} 


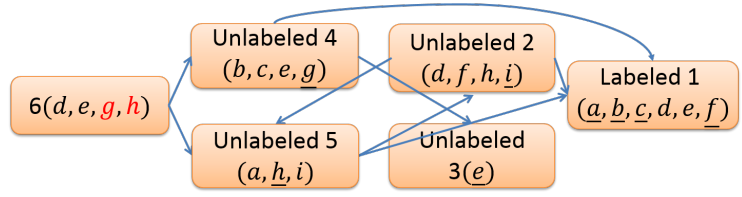

Figure 1: The trading graph $T G(6,\{g, h\},[5]), \underline{h}$ denotes $h$ is owned currently by the agent. The common order is $a \succ b \succ c \succ d \succ e \succ f \succ g \succ h \succ i$.

allocated subsets of objects from their preference list. When an agent is allocated a subset of objects we say that he owns these objects. Let $S \subseteq N$ and suppose that some of the agents in $S$ have been allocated some objects and the allocated objects to each agent appear in the same indifference class of this agent. At any time during the execution of the algorithm, each agent who is allocated more than one object is called labeled and unlabeled otherwise. Let $i \in N$, and let $B \subseteq L(i)$ be such that $i$ is not allocated any object in $B$. The trading graph $(T G)$ is a directed graph $T G(i, B, S)$ with $\{i\} \cup S$ as the set of nodes, and arcs defined as follows: Let agent $i$ point to any agent in $S$ who owns any object in $B$. For each unlabeled agent, e.g., $j \in S$, to which $i$ points, suppose the current object allocated to $j$ is in $j$ 's $k$ th indifference class $C_{k}^{j}$. Then let $j$ point to any agent in $S$ who currently owns any object in $C_{k}^{j}$ not owned by $j$. Continue this process for the new pointed-to and unlabeled agents until no agent in $S$ needs to point to the other agents. See Figure 1 for how $T G(6,\{g, h\},[5])$ is constructed: agent 6 points to agent 4 and 5 since currently agent 4 owns $g$ and agent 5 owns $h$; then, as agent 4 is unlabeled, agent 4 points to agents 3 and 1 since agent 3 owns $e$ and agent 1 owns $b$ and $c$; similarly, agent 5 points to agents 1 and 2; agent 1 is labeled so only agent 2 points to agents 1 and 5 .

Let $H=\{a \in L(i) \mid$ there is a (directed) path from $i$ to a labeled agent in $T G(i, a, S)\}$. Note that, as labeled agents do not point to any agents, no intermediate agent on a directed path is labeled. Note that $H$ may be empty, and it can be found by breadth first search (BFS). If $H \neq \emptyset$, let $\ell$ be the highest indifference class of $i$ with $H \cap C_{\ell}^{i} \neq \emptyset$. Define $\max T G(i, L(i), S)$ to be the highest order object in $H \cap C_{\ell}^{i}$ (e.g., in Fig. 1, $\max T G(6,\{d, e, g, h\},[5])=\{g\})$. If $\max T G(i, L(i), S) \neq \emptyset$, then there is a path from $i$ to a labeled agent in $T G(i, a, S)$, which can be found by BFS. Suppose the path is $\left(i_{0}, i_{1}, i_{2}, \cdots, i_{k}\right)$, where $i_{0}=i$ and only $i_{k}$ is labeled. Now denote $\operatorname{Trading}(i, a, S)$ to be a procedure that allocates the object owned by $i_{s+1}$ to $i_{s}$, for $s=0,1, \cdots, k-1$. Note that $i_{k}$ may own more than one object for which $i_{k-1}$ has pointed to $i_{k}$. In this case, the highest order object among such objects is allocated to $i_{k-1}$. After trading, if $i_{k}$ still owns more than one object, keep $i_{k}$ labeled and unlabel $i_{k}$ otherwise. In Fig. 1, Trading $(6, g,[5])$ allocates $g$ to agent 6 and $b$ to agent 4 , since $b \succ c$, and keeps agent 1 labeled. Note that $C_{n_{2}+1}^{i}=\emptyset, \forall i \in\left[n_{1}\right]$. With these preliminaries, we present our algorithm SDMT-2 (Algorithm 2). In the following, we will refer to $k$ th iteration of the "for loop" in SDMT-2 as $k$ th loop. Observe that in the $k$ th loop, $j_{1}$ is the highest indifference class of $i$ where $i$ can obtain unallocated objects, and $j_{2}$ is the highest indifference class of $i$ where $i$ can obtain objects from the allocated objects without hurting the agents prior to $i$.

Observation 4.1. For each agent $i$, after $i$ 's turn in "for loop" of SDMT-2, if $i$ is allocated no object, then he will be allocated no object when SDMT-2 terminates. Otherwise, if $i$ is provisionally allocated some objects in his turn, then in the final matching he will be allocated an object in the same indifference class as his initially allocated objects. 


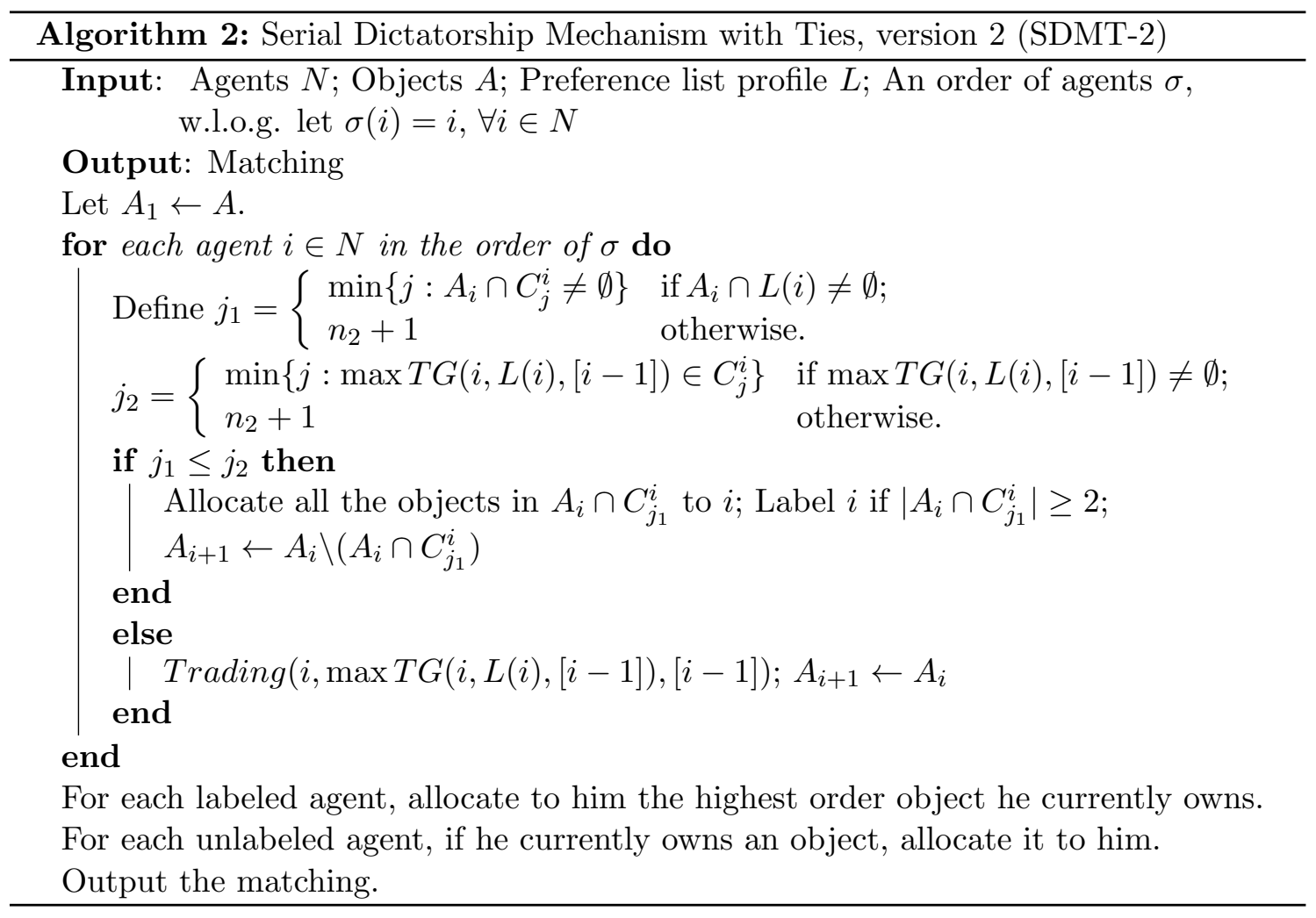

Observation 4.2. For each agent $i$, after $i$ 's turn, if $i$ is allocated an object $a \in C_{j}^{i}$, then all the objects in $\cup_{k=1}^{j} C_{k}^{i}$ have been allocated to either $i$ or to some agents prior to $i$. Once an object is allocated, it remains allocated until the end of the for loop.

Now we establish the equivalence of SDMT-1 and SDMT-2.

Theorem 4.3. Given the same input, SDMT-1 and SDMT-2 match the same set of agents. Furthermore, for each matched agent $i$, the object allocated to $i$ in SDMT-1 is in the same indifference class of $i$ as the object allocated to him in SDMT-2.

It is easy to see that both SDMT-1 and SDMT-2 reduce to SDM if all agents have strict preference over objects. We say an agent (respectively, an object) is frozen if the allocation of this agent (respectively, object) remains the same until the end of SDMT-2.

Theorem 4.4. SDMT-2 is truthful, Pareto optimal, and terminates in $O\left(n_{1}^{2} \gamma\right)$ running time.

\subsection{Randomized mechanism}

We now present a universally truthful and Pareto optimal mechanism with approximation ratio of $\frac{e}{e-1}$, where agents may have weights and their preferences may involve ties (see Algorithm 3, where $g(y)=e^{y-1}$ ). When preferences are strict, Algorithm 3 reduces to a variant of RSDM that has been used in weighted online bipartite matching with approximation ratio $\frac{e}{e-1}$ (see [3] and [15]). Our analysis of Algorithm 3 is a non-trivial extension of the primal-dual analysis from [15] to the case where agents' preferences may involve ties.

To gain some high-level intuition behind this extension, we highlight here the similarities and differences between our problem and that of online bipartite matching. Our problem with strict preferences and without weights is closely related to online bipartite 


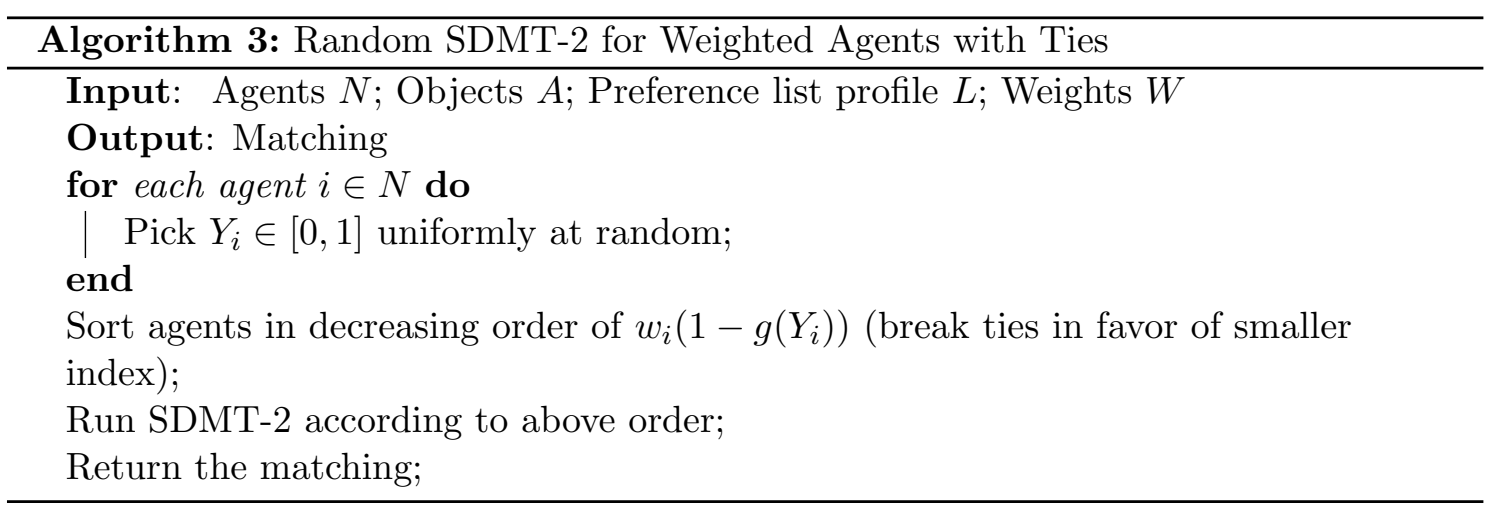

matching. ${ }^{6}$ If each agent in our problem ranks his desired objects in the order that precisely follows the arrival order of objects in the online bipartite matching, the two problems are equivalent. Therefore, we extend the analysis of this particular setting, where each agent's preference list is a sublist of a global preference list, to the general case where agents preferences are not constrained and may involve ties, and furthermore agents may have weights.

If the weights are public, Algorithm 3 is universally truthful and Pareto optimal. This is because it chooses a random order of the agents, given the weights, and then runs SDMT-2 according to this order. It follows from SDMT-2 that, if the order of the other agents is given, an agent can get a better object if he appears earlier in this order. Then it is not difficult to see that if the weights are private, and under the assumption that no agent is allowed to bid over his private weight, Algorithm 3 is still universally truthful in the sense that no agent will lie about his preferences or weight.

Theorem 4.5. Algorithm 3 is universally truthful, even if the weights and preference lists of the agents are their private knowledge, assuming that no agent can over-bid his weight.

\section{Analysis of the approximation ratio}

To analyze the approximation ratio of Algorithm 3, we first write the LP formulation of the (relaxed) problem and its dual LP formulation. Given random variables $Y_{i}$, we will define a primal solution and a dual solution obtained by Algorithm 3, which are both random variables, such that the objective value of the primal solution is always at least a faction $F$ of the objective value of the dual solution, and that the expectation of duals is feasible. Hence, the expectation of the primal solution is at least $F$ times the expectation of duals, which by weak LP duality, is at least optimal value of the primal LP. The standard LP and its dual of our problem are given in Figure 2.

By Lemma 5.1, proved in [15], the inverse of approximation ratio is $F \in[0,1]$.

Lemma 5.1 ([15]). Suppose that a randomized primal-dual algorithm has a primal feasible solution with value $P$ (which is a random variable) and a dual solution which is not necessarily feasible, with value $D$ (which is also a random variable) such that

1. for some universal constant $F, P \geq F \cdot D$, always, and

\footnotetext{
${ }^{6}$ In the online bipartite matching problem [8], vertices of one partition (think of them as agents) are given and fixed, while vertices of the other partition (think of them as objects) arrive in an adversarial order. When an item arrives, we get to see the incident edges on agents. These edges indicate the set of agents that desire this object. The algorithm must immediately match this object to one of the unmatched agents desiring it (or choose to throw it away). In the end, the size of the obtained matching is compared with the optimum matching in the realized graph.
} 


$$
\begin{gathered}
\max \sum_{(i, a) \in E} w_{i} x_{i a} \quad \text { s.t. } \forall i \in N: \sum_{a:(i, a) \in E} x_{i a} \leq 1 ; \quad \forall a \in A: \sum_{i:(i, a) \in E} x_{i a} \leq 1 ; \\
\forall(i, a) \in E: \quad x_{i a} \geq 0 .
\end{gathered}
$$

$\min \sum_{i \in N} \alpha_{i}+\sum_{a \in A} \beta_{a} \quad$ s.t. $\forall(i, a) \in E: \alpha_{i}+\beta_{a} \geq w_{i} ; \quad \forall i \in N, a \in A: \alpha_{i}, \beta_{a} \geq 0$.

Figure 2: The primal and dual LP relaxation. $G=(V, E)$, where $V=N \cup A$ and $E=\{(i, a), i \in N, a \in A\}$

\section{2. the expectation of the randomized dual variables form a feasible dual solution.}

The expectation of $P$ is then at least F. OPT where OPT is the value of the optimum solution.

Note that in Lemma 5.1, OPT is the value of maximum weighted matching, which is no less than the value of maximum weighted Pareto optimal matching. Hence, if the condition of Lemma 5.1 holds, the approximation ratio of the mechanism is $\frac{1}{F}$. The construction of the duals depends on function $g$. Let $F=\left(1-\frac{1}{e}\right)$. It is not difficult to see that

$$
\forall \theta \in[0,1] \int_{0}^{\theta} g(y) d y+1-g(\theta) \geq F
$$

For any random selection of $Y_{i}, i \in N$, let $\vec{Y}=\left(Y_{1}, Y_{2}, \cdots, Y_{n_{1}}\right)=\left(Y_{i}, Y_{-i}\right)$. Following the procedure of Algorithm 3, whenever agent $i$ is matched to object $a$, let

$$
x_{i a}(\vec{Y})=1, \quad \alpha_{i}(\vec{Y})=w_{i} g\left(Y_{i}\right) / F, \quad \beta_{a}(\vec{Y})=w_{i}\left(1-g\left(Y_{i}\right)\right) / F .
$$

For all unmatched $i$ and $a$, set $x_{i a}(\vec{Y})=\alpha_{i}(\vec{Y})=\beta_{a}(\vec{Y})=0$. By this definition, it follows that for any $Y_{i}, i \in N$, the random value $P$ of primal solution $\left\{x_{i a}(\vec{Y}), i \in N, a \in A\right\}$ is always at least $F \cdot D$, where $D$ is random value of the dual solution $\left\{\alpha_{i}(\vec{Y}), i \in N, \beta_{a}(\vec{Y}), a \in\right.$ $A\}$.

Hence, to satisfy the conditions of Lemma 5.1, we need to show that the expectation of dual solution $\left\{\alpha_{i}(\vec{Y}), i \in N, \beta_{a}(\vec{Y}), a \in A\right\}$ is feasible for the dual LP, implying that the approximation ratio of Algorithm 3 is at most $\frac{1}{F}=\frac{e}{e-1}$. The main technical difficulty lies in proving the dominance lemma and the monotonicity lemma (see Lemma 5.3 and 5.4). To prove these two lemmas, we define a threshold which specifies whether an agent still gets matched if he is put back into the order after he is first removed from it. For an agent with strict preferences, such a threshold is the same as that of defined in the online bipartite matching problem. However, in the presence of ties, the same defined threshold does not work. We show how to define such a threshold for our algorithm.

Fix an agent $i \in\left[n_{1}\right]$ and object $a \in A$, such that $(i, a) \in E$. Fix $Y_{-i}$, the random variables $Y_{i^{\prime}}$ for all other agents $i^{\prime} \neq i$. We use $\sigma$ to denote the order of agents under $Y_{-i}$, e.g., $\sigma(1)$ is the first agent, etc, and $\sigma([i])=\{\sigma(1), \sigma(2), \cdots, \sigma(i)\}$. Consider Algorithm 3 running on the instance without agent $i$ and let us denote this procedure by $A L G_{-i}$, where $\sigma$ is the order of agents under $A L G_{-i}$. The threshold $y^{c}$ is then defined as follows:

1. If $a$ is unmatched in $A L G_{-i}$, let $y^{c}=1$.

2. Otherwise, suppose that $a$ is matched in $A L G_{-i}$ to some agent $i^{\prime}$. Then consider the allocations just after the for loop in $A L G_{-i}$ terminated.

If $i^{\prime}$ is labeled, set $y^{c}=1$. 
3. Otherwise, suppose $a \in C_{j}^{i^{\prime}}$ and construct the trading graph $T G\left(i^{\prime}, C_{j}^{i^{\prime}} \backslash\{a\},\left[n_{1}\right] \backslash\{i\}\right)$ from all the objects in $C_{j}^{i^{\prime}}$ other than $a$ (note that $\sigma\left(\left[n_{1}-1\right]\right)=\left[n_{1}\right] \backslash\{i\}$ ).

If there is a path in $T G\left(i^{\prime}, C_{j}^{i^{\prime}} \backslash\{a\},\left[n_{1}\right] \backslash\{i\}\right)$ from $i^{\prime}$ to a labeled agent, set $y^{c}=1$.

4. Otherwise, define

$i^{\prime \prime}=\arg \min \left\{w_{l}\left(1-g\left(Y_{l}\right)\right) \mid\right.$ there is a path from $i^{\prime}$ to $\left.l \operatorname{in} T G\left(i^{\prime}, C_{j}^{i^{\prime}} \backslash\{a\},\left[n_{1}\right] \backslash\{i\}\right)\right\}$. If $w_{i}(1-g(y))=w_{i^{\prime \prime}}\left(1-g\left(Y_{i^{\prime \prime}}\right)\right)$ has a solution $y \in[0,1]$ define $y^{c}$ to be this solution. $(g(y)$ is strictly increasing so if there is a solution, it is unique)

5. Otherwise define $y^{c}$ to be 0 .

Now consider Algorithm 3 running on the original instance (denote such procedure as $A L G)$, with $\left(Y_{i}, Y_{-i}\right)$ fixed. Suppose that $\tau$ is the order of agents under this execution of $A L G$. The intuition behind the definition of $y^{c}$ is the following. Having $Y_{-i}$ fixed, we want to define $y^{c}$ such that if we run $A L G$ with $\left(Y_{i}, Y_{-i}\right)$ where $Y_{i}<y^{c}$, then agent $i$ gets matched (to any of his objects). If (1) holds, then $Y_{i}<1$ and $i$ will be matched because at least object $a$ is his available candidate. If (2) happens, then $Y_{i}<1$ and $i$ will also be matched because object $a$ can be re-allocated from the labeled agent $i^{\prime}$ to $i$. Case (3) is analogous to (2) with the only difference that we now have a trading path from $i^{\prime}$ to a labeled agent. Case (4) will be discussed just after Observation 5.2.

Observation 5.2. Assume that agent $i$ is unmatched in his turn in the "for loop" of $A L G$. Suppose $\tau(u)=i$, which means $i$ selects his object in u'th iteration of the "for loop". Then at end of $k$ 'th iteration of the "for loop", $k \geq u$, there is no path from $i$ to a labeled agent in $T G(i, L(i), \tau([k]))$ (meaning this graph is frozen).

Proof. By SDMT-2, we know $A_{u} \cap L(\tau(u))=A_{u} \cap L(i)=\emptyset$, which means all the objects in $L(i)$ have been allocated to agents $\tau([u-1])$. Since $\tau(u)$ is unmatched, there is no path from $\tau(u)$ to any labeled agent in $T G(\tau(u), L(\tau(u)), \tau([u-1]))$. Let $S \subseteq \tau([u-1])$ be the set reachable from $\tau(u)$ in $T G(\tau(u), L(\tau(u)), \tau([u-1]))$, clearly, each agent in $S$ is unlabeled. Actually any agent in $S$ is frozen. Therefore, any path through $i$ after $u$ 'th iteration will reach an unlabeled agent.

The following two properties (dominance and monotonicity) are well known for agents with strict preference orderings. We generalize them to agents with indifference. The difficulty of proving both dominance and monotonicity lemmas (Lemma 5.3 and 5.4) lies in case (4). This is our main technical contribution as compared to the analysis in [15].

Recall that $\tau$ ( $\sigma$, resp.) is the order of agents under the execution of $A L G\left(A L G_{-i}\right.$, resp.). We first deal with the case (4) of the dominance lemma. Note that in this case there is a path from $i^{\prime}$ to $i^{\prime \prime}$ in $T G\left(i^{\prime}, C_{j}^{i^{\prime}} \backslash\{a\},\left[n_{1}\right] \backslash\{i\}\right)$ and agent $i^{\prime \prime}$ is unlabeled. We will prove dominance by contradiction, using the following two main steps. Suppose $\sigma(u)=i^{\prime \prime}$ in $A L G_{-i}$, then $\tau(u+1)=i^{\prime \prime}$ in $A L G$ under case (4). Suppose that $Y_{i}<y^{c}$ and $i$ is not matched in $A L G$, then the outcome of $A L G$ is the same as that of $A L G_{-i}$ for all the other agents except $i$. Based on this fact, first, we prove that either $i^{\prime}$ is labeled or there is a path, call it $P_{1}$, from $i^{\prime}$ to a labeled agent in $T G\left(i^{\prime}, C_{j}^{i^{\prime}}, \tau([u])\right)$ at the end of the $u$ th iteration of the for loop in $A L G$. Second, due to the above property, we argue that there is a path, say $P_{2}$, from $i$ to a labeled agent in $T G(i, a, \tau([u]))$ at the end of the $u$ th iteration of the for loop in $A L G$, contradicting Observation 5.2; thus $i$ will be matched. Path $P_{2}$ is constructed by the concatenation of $\operatorname{arc}\left(i, i^{\prime}\right)$ and path $P_{1}$, or the concatenation of arc $\left(i, i^{\prime \prime \prime}\right)$, for some $i^{\prime \prime \prime}$ on path $P_{1}$, and the rest of path $P_{1}$. The existence of $P_{1}$ is proved by a careful analysis of the structure of frozen subgraphs of the trading graph as the algorithm proceeds; the details can be found in the proof of Lemma 5.3. 
Lemma 5.3 (DOMINANCE LEMMA). Given $Y_{-i}$, i gets matched if $Y_{i}<y^{c}$.

Proof. (short version) Case 1: If $a$ is unmatched in $A L G_{-i}$ and $i$ is unmatched in $A L G$, then $a$ is available to agent $i$ in $A L G$. Contradiction. Hence, $i$ will be matched.

Case 2: If $a$ is matched to $i^{\prime}$ in $A L G_{-i}$ :

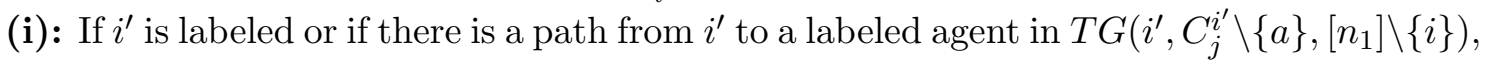
if $i$ is unmatched, then there is a path from $i$ to a labeled agent in $T G\left(i, a,\left[n_{1}\right]\right)$, then by Trading $\left(i, a,\left[n_{1}\right]\right)$, we get a Pareto improvement, contradicting that SDMT-2 is Pareto optimal.

(ii): The case $y^{c}=0$ is trivial. Suppose that $\sigma(u)=i^{\prime \prime}$ in $A L G_{-i}$, then if $Y_{i}<y^{c}$, we know $w_{i}\left(1-g\left(Y_{i}\right)\right)>w_{i^{\prime \prime}}\left(1-g\left(Y_{i^{\prime \prime}}\right)\right)$ meaning the agent $i$ is prior to agent $i^{\prime \prime}$ in $A L G$. Then $\tau(u+1)=i^{\prime \prime}$ in $A L G$. If $i$ is unmatched in $A L G$, then procedure $A L G$ is the same as $A L G_{-i}$ for all the other agents except $i$. The case $i^{\prime \prime}=i^{\prime}$ or $a \in A_{u+1}$ is similar to above argument. we only consider $a \notin A_{u+1}$ and $i^{\prime \prime} \neq i^{\prime}$. We will show that in the $(u+1)$ st iteration of for loop in $A L G, \tau(u+1)$ gets object $b$ via the trading graph.

Thus, at the end of $u$ th iteration of the for loop of $A L G$, if $B$ is the set of objects allocated to $i^{\prime}$, we have three cases (noting $A L G$ is the same as $A L G_{-i}$ ) for all the other agents except $i$ :

Case (ii-1): $i^{\prime}$ is labeled, then $a \in B$. Otherwise, if $a \notin B$, then in $(u+1)$ st for loop iteration of $A L G, a$ will not be allocated to $i^{\prime}$ at the end this $(u+1)$ st iteration by SDMT-2. Thus $a \in B$.

Case (ii-2): $i^{\prime}$ is unlabeled and $B=\{a\}$, then there is a path from $i^{\prime}$ to a labeled agent in $T G\left(i^{\prime}, C_{j}^{i^{\prime}} \backslash\{a\}, \tau([u])\right)$. Otherwise, all agents reachable from $i^{\prime}$ are frozen after $u$ th iteration, i.e., their allocations are fixed, since all the objects in their indifference class where they are allocated to some objects were allocated by Observation 4.2. Thus, $T G\left(i^{\prime}, C_{j}^{i^{\prime}} \backslash\{a\}, \tau([u])\right)=T G\left(i^{\prime}, C_{j}^{i^{\prime}} \backslash\{a\}, \tau([u+1])\right)$. Now, $\tau(u+1)$ is reachable from $i^{\prime}$ in $T G\left(i^{\prime}, C_{j}^{i^{\prime}} \backslash\{a\}, \tau([u+1])\right)$ while $\tau(u+1)$ does not appear in $T G\left(i^{\prime}, C_{j}^{i^{\prime}} \backslash\{a\}, \tau([u])\right)$; contradiction.

Case (ii-3): $i^{\prime}$ is unlabeled and $B=\{c\}$, where $c \neq a$. Then there is a path from $i^{\prime}$ to a labeled agent in $T G\left(i^{\prime},\{a\}, \tau([u])\right)$. Otherwise $a$ and the agent matched to $a$ is frozen at the end of $u$ th loop in $A L G$, i.e., $a$ will not be matched to $i^{\prime}$ at the end of for loop of $A L G$; contradiction.

Thus, in each of these three cases, there is a path from $i$ to a labeled agent in $T G(i, a, \tau([u]))$ at the end of $u$ th for loop iteration in $A L G$, contradicting Observation 5.2. Hence, $i$ must be matched.

Let $\beta_{a}(\vec{Y})$ be $\beta_{a}^{s}$ when $A L G$ is the procedure of Algorithm 3 running on the original instance when $Y_{-i}$ is fixed and $Y_{i}=s$, i.e., $\beta_{a}^{s}=\beta_{a}\left(\left(s, Y_{-i}\right)\right)$. Let $\beta_{a}^{c}=\beta_{a}^{y^{c}}=w_{i}(1-$ $\left.g\left(y^{c}\right)\right) / F$.

The difficulty of the proof of the monotonicity lemma (Lemma 5.4) still lies in case (4). We prove it in three steps. Recall that $\tau$ ( $\sigma$, respectively) is the order of agents under the execution of $A L G$ ( $A L G_{-i}$, respectively). Let $\sigma(u)=i^{\prime \prime}$ in $A L G_{-i}$. Observe that the monotonicity lemma means that $a$ is allocated to an agent prior to $i^{\prime \prime}$ or $i^{\prime \prime}$. The proof of this is easy in the case where $Y_{i}>y^{c}$. To see this, note that $A L G$ and $A L G_{-i}$ result in the same tentative allocation at the end of their $u$ th loop, since $i$ is inserted back after $i^{\prime \prime}$. Hence, we only need to consider the case where $Y_{i}<y^{c}$, which implies that $i$ is inserted back prior to $i^{\prime \prime}$. First, we prove that no agent, except $i$, is allocated a better object in $A L G$ compared to $A L G_{-i}$. The argument is by contradiction: suppose there exists an agent $i^{\prime \prime \prime}$ who receives a better object in $A L G$ than in $A L G_{-i}$. Then $i$ must be inserted 
before $i^{\prime \prime \prime}$. Consequently, there exists an agent $s$ prior to $i^{\prime \prime \prime}$ who will get a worse object in $A L G$ than in $A L G_{-i}$. Based on this fact, and using an alternating path argument, it is proved that there exists a path from $s$ to $i^{\prime \prime \prime}$ in $s$ 's trading graph constructed from a higher indifference class of $s$ (than $s$ 's allocated indifference class in $A L G$ ) after $i^{\prime \prime \prime}$ is allocated in $A L G$. This contradicts the fact that this path should not exist since the graph from that higher indifference class is frozen. Secondly, if $i^{\prime}$ gets a worse object in $A L G$ compared to $A L G_{-i}$, we prove that $a$ must be allocated to an agent prior to $i^{\prime}$, which is in turn prior to $i^{\prime \prime}$. The reason is as follows: by Observation 4.2, a must be allocated and frozen before $i^{\prime}$ is considered in $A L G$. Thirdly, if $i^{\prime}$ gets an object in $A L G$ in the same indifference class as $a$, then we prove that there exists an agent $s^{*}$ prior to $i^{\prime \prime}$, and suppose $\tau\left(u^{*}\right)=s^{*}$, such that there is a path from $s^{*}$ to $i^{\prime}$ in $T G\left(s^{*}, C_{j^{*}}^{s^{*}}, \tau\left(\left[u^{*}\right]\right)\right)$ at the end of the $u^{*}$ th loop of $A L G$, where $C_{j^{*}}^{s^{*}}$ is the indifference class in which $s^{*}$ is allocated an object in $A L G_{-i}$. As a consequence, by Observation 4.2, $a$ is allocated to an agent prior to $s^{*}$ and all the agents reachable from $s^{*}$ in $T G\left(s^{*}, C_{j^{*}}^{s^{*}}, \tau\left(\left[u^{*}\right]\right)\right)$ are frozen, then $a$ will be finally allocated to an agent prior to $s^{*}$ in $A L G$. This means that $a$ is allocated to an agent prior to $i^{\prime \prime}$. This gives the monotonicity lemma, which together with dominance is used to prove Lemma 5.5 .

Lemma 5.4 (MONOTONICITY LEMMA). Given $Y_{-i}$, for all choices of $Y_{i}, \beta_{a}^{Y_{i}} \geq \beta_{a}^{c}$.

Lemma $5.5([15]) . \forall(i, a) \in E, \mathbb{E}_{\vec{Y}}\left(\alpha_{i}(\vec{Y})+\beta_{a}(\vec{Y})\right) \geq w_{i}$.

From Lemma 5.1 and Lemma 5.5, we have the following theorem.

Theorem 5.6. Algorithm 3 achieves approximation ratio of $\frac{e}{e-1}$ for weighted agents with indifference.

\section{Lower bounds}

Preliminaries. We will use Yao's minmax principle [31] to obtain a non-trivial lower bound for universally truthful and Pareto optimal mechanisms and another lower bound for an "online" version of our problem. We first need some preliminaries.

Let us fix the number of agents $n_{1}$ and the number of objects $n_{2}$. The number of distinct instances and the number of deterministic truthful and Pareto optimal mechanisms are finite. Denote by $\mathcal{T}$ the set of deterministic truthful and Pareto optimal mechanisms with input size $n_{1}$ and $n_{2}$, and $\mathcal{I}$ the set of instances with input size $n_{1}$ and $n_{2}$. Let $\mathrm{P}$ and $\mathrm{Q}$ denote the set of probability distributions on $\mathcal{T}$ and $\mathcal{I}$, respectively. Denote $\mathbb{E}_{p, q}\left(r\left(T_{p}, I_{q}\right)\right)$ as the inverse of approximation ratio when the input distribution is $q \in \mathrm{Q}$ on $\mathcal{I}$ and a universally truthful mechanism and Pareto optimal mechanism $T_{p}$ taken from $\mathcal{T}$ with probability $p \in \mathrm{P}$. Then the minmax theorem [31] states the following:

$$
\min _{q \in \mathrm{Q}} \max _{p \in \mathrm{P}} \mathbb{E}_{p, q}\left(r\left(T_{p}, I_{q}\right)\right)=\max _{p \in \mathrm{P}} \min _{q \in \mathrm{Q}} \mathbb{E}_{p, q}\left(r\left(T_{p}, I_{q}\right)\right)
$$

and

$$
\min _{q \in \mathrm{Q}} \max _{T \in \mathcal{T}} \mathbb{E}_{q}\left(r\left(T, I_{q}\right)\right)=\max _{p \in \mathrm{P}} \min _{I \in \mathcal{I}} \mathbb{E}_{p}\left(r\left(T_{p}, I\right)\right) .
$$

As a consequence, for any $q \in \mathrm{Q}$ and $p \in \mathrm{P}$, we have

$$
\max _{T \in \mathcal{T}} \mathbb{E}_{q}\left(r\left(T, I_{q}\right)\right) \geq \min _{I \in \mathcal{I}} \mathbb{E}_{p}\left(r\left(T_{p}, I\right)\right) .
$$

This inequality states that an upper bound on the inverse of the approximation ratio of the best universally truthful and Pareto optimal mechanism $T_{p}$ on the worst instance 
is upper bounded by the inverse of the approximation ratio of the best deterministic truthful and Pareto optimal mechanism on any random instances. Hence, in order to bound $\min _{I \in \mathcal{I}} \mathbb{E}_{p}\left(r\left(T_{p}, I\right)\right)$, we only need to construct an appropriate random instance and show the upper bound of the best deterministic truthful and Pareto optimal mechanism on this random instance. Consider the triangle instance where $N=\left\{1,2, \cdots, n_{1}\right\}$ and $A=\left\{a_{1}, a_{2}, \cdots, a_{n_{1}}\right\}$, and an agent $i$ 's preference ordering is $a_{1} \succ_{i} a_{2} \succ_{i} \cdots \succ_{i} a_{i}$, for any $i \in N$.

Let $\mathcal{S}$ be the set of all the permutations of agents' preference lists of the triangle instance. Consider now a random instance $S_{u n i}$ as the uniform distribution of $\mathcal{S}$. It is obvious that the output of any serial dictatorship mechanism (which is deterministic truthful and Pareto optimal) running on $\mathcal{S}$ is the same. Hence, for any serial dictatorship mechanism $(\mathrm{SDM}), \mathbb{E}_{\text {uni }}\left(r\left(S D M, S_{\text {uni }}\right)\right)$ is equal to the inverse of the approximation ratio of RSDM (which is just SDM with uniformly random order of agents) when running on the triangle instance.

Online lower bound. We now apply these preliminaries to the online version of our problem. Recall that applicants in this online problem are truthful due to truthfulness of serial dictatorship mechanism. The strategy of the administrator is a random order in which the applicants are interviewed. More precisely, let $\Pi$ denote the set of all the permutations of applicants and $P$ be the set of probability distributions on $\Pi$. Let $\Pi_{p}$ be a random order of applicants, where the order is selected according to the distribution $p \in P$ on $\Pi$, and then the strategy set of the administrator is $\left\{\Pi_{p}, p \in P\right\}$. We will show that the best strategy for the administrator is to select applicants' order uniformly at random.

Theorem 6.1. The best strategy for the administrator in the online problem is to select applicants' order uniformly at random. Thus, any other randomized strategy, than the one used in Algorithm 3, would lead to an approximation guarantee worse than $\frac{e}{e-1}$.

Proof. Let $\mathbb{E}_{p, q}\left(r\left(\Pi_{p}, I_{q}\right)\right)$ be the inverse of the approximation ratio when the random order is $\Pi_{p}$ and the random instance is $I_{q}$, and $\Pi_{u n i}$ denotes the uniform order. By the approximation ratio of $\mathrm{RSDM}$, for any $I, \mathbb{E}_{\text {uni }}\left(r\left(\Pi_{\text {uni }}, I\right)\right) \geq \frac{e-1}{e}$. Now for upper bound of $\mathbb{E}_{p, q}\left(r\left(\Pi_{p}, I_{q}\right)\right)$, by Yao's principle, $\max _{T \in \Pi} \mathbb{E}_{q}\left(r\left(T, I_{q}\right)\right) \geq \min _{I \in \mathcal{I}} \mathbb{E}_{p}\left(r\left(T_{p}, I\right)\right)$. Recall that $S_{\text {uni }}$ is the uniform distribution over $\mathcal{S}$. Then we need to upper bound $\max _{T \in \Pi} \mathbb{E}_{q}\left(r\left(T, S_{\text {uni }}\right)\right)$, which in fact is equal to the inverse of the approximation ratio obtained by running RSDM on the triangle instance, which is $\frac{e-1}{e}$. The argument is as follows. Suppose object $a_{k}$ is allocated by RSDM with probability $p_{k} \leq 1$ on the triangle instance. Then, because there are $n_{1}-k+1$ agents with $a_{k}$ in their preference lists, each such agent obtains $a_{k}$ with equal probability $\frac{p_{k}}{n_{1}-k+1}$. Therefore, agent $i$ is allocated an object with probability $\sum_{j=1}^{i} \frac{p_{j}}{n_{1}-j+1}$, which is at most $\min \left\{1, \sum_{j=1}^{i} \frac{1}{n_{1}-j+1}\right\}$. Now, summing over all the agents, by a simple calculation we get that the expected cardinality of allocated agents is at most $n_{1}\left(1-\frac{1}{e}\right)$, for large enough $n_{1}$. Hence, the approximation ratio is tight.

Lower bound for randomized mechanisms. If we can prove that the output of any deterministic truthful and Pareto optimal mechanism running on $\mathcal{S}$ is the same as that of a serial dictatorship mechanism, then $\max _{T \in \mathcal{T}} \mathbb{E}_{q}(r(T, I))=1-\frac{1}{e}$. To show our lower bound it suffices to show that the sum of the sizes of all the matchings output by any deterministic truthful and Pareto optimal mechanism that runs on $\mathcal{S}$ is smaller than that of any serial dictatorship run on $\mathcal{S}$. Then $\max _{T \in \mathcal{T}} \mathbb{E}_{q}(r(T, I))=1-\frac{1}{e}$. We use $\#^{\phi}(\mathcal{S})$ to denote the sum of sizes of all the matchings output by mechanism $\phi$ when run on $\mathcal{S}$. Recall that SDM denotes a serial dictatorship, then we would like to prove that $\#^{\phi}(\mathcal{S}) \leq \#^{S D M}(\mathcal{S})$, for any $n_{1}$ and $n_{2}$ and for any universally truthful and Pareto optimal mechanism $\phi$. Until 
now, we can prove this inequality assuming $n_{1}=n_{2}=3$, which gives the lower bound of $\frac{18}{13}$ for any universally truthful and Pareto optimal mechanism.

Theorem 6.2. For any deterministic truthful and Pareto optimal mechanism $\phi, \#^{\phi}(\mathcal{S}) \leq$ 13 , when $n_{1}=3$. Thus, any universally truthful and Pareto optimal mechanism for this problem has an approximation ratio of at least $\frac{18}{13}$.

Note that Theorem 6.2 shows that $\min _{I \in \mathcal{I}} \mathbb{E}_{p}\left(r\left(T_{p}, I\right)\right) \leq \max _{T \in \mathcal{T}} \mathbb{E}_{q}\left(r\left(T, S_{\text {uni }}\right)\right) \leq \frac{13}{18}$, for any $p \in \mathrm{P}$, and $S_{\text {uni }}$ is the uniform distribution over $\mathcal{S}$. Hence, the approximation ratio is at least $\frac{18}{13}$.

Lower bound for non-bossy mechanisms. We first briefly define the concept of nonbossiness for a deterministic mechanism (see, e.g.,[23]). A deterministic mechanism $\phi$ is non bossy, if for any strict preference list $L(i), L^{\prime}(i), L(-i)$ and $i \in N$, if $\phi_{i}(L(i), L(-i))=$ $\phi_{i}\left(L^{\prime}(i), L(-i)\right)$ then $\phi(L(i), L(-i))=\phi\left(L^{\prime}(i), L(-i)\right)$. Pápai [23] proved that nonbossiness with truthfulness is equivalent to group truthfulness. Pycia and Ünver [25] characterized all the mechanisms which are group strategy-proof (note, strategy-proofness is a synonym with truthfulness) and Pareto optimal by trading cycle with owners and brokers mechanisms. Bade [7] showed that any mechanism which is group strategy-proof and Pareto optimal is $r$-equivalent to serial dictatorship, in the sense that if the order of agents is generated uniformly at random, the resulting random matching from serial dictatorship mechanism is the same as that from any group strategy-proof and Pareto optimal mechanism. Hence, by arguments in the preliminaries that use Yao's principle, we have the following tight lower bound for any group strategy-proof and Pareto optimal mechanism.

Theorem 6.3. No universally truthful, non-bossy and Pareto optimal mechanism can achieve the approximation ratio better than $\frac{e}{e-1}$.

Note that our mechanism with strict preference list and weights is universally truthful, nonbossy and Pareto optimal.

\section{Conclusion}

Whilst this paper has focused on Pareto optimality in the HA context, stronger forms of optimality are possible. For example, minimum cost (or maximum utility), rank-maximal and popular matchings can also be studied in the HA context, and a matching of each of these types is Pareto optimal (see, e.g., [22, Sec. 1.5] for definitions). As Pareto optimality is a unifying feature of all of these other forms of optimality, we chose to concentrate on this concept in our search for randomized truthful mechanisms that can provide good approximations to maximum matchings with desirable properties.

Note that the lower bound on the performance of deterministic truthful mechanisms that produce Pareto optimal matchigns extends to those producing matchings that satisfy these stronger optimality criteria. It will thus be the focus of future work to consider the performance of randomized truthful mechanisms for these problems.

\section{Acknowledgements}

We would like to thank Anna Bogomolnaia and Hervé Moulin for helpful discussions concerning the results in this paper. We would also like to thank anonymous reviewers for their valuable remarks on this paper. 


\section{References}

[1] A. Abdulkadiroğlu and T. Sönmez. Random serial dictatorship and the core from random endowments in house allocation problems. Econometrica, 66(3):689-701, 1998.

[2] D.J. Abraham, K. Cechlárová, D.F. Manlove, and K. Mehlhorn. Pareto optimality in house allocation problems. In Proc. ISAAC '04, volume 3341 of $L N C S$, pages 3-15. Springer, 2004.

[3] G. Aggarwal, G. Goel, C. Karande, and A. Mehta. Online vertex-weighted bipartite matching and single-bid budgeted allocations. In Proc. SODA '11, pages 1253-1264. ACM-SIAM, 2011.

[4] H. Aziz, F. Brandt, and P. Harrenstein. Pareto optimality in coalition formation. Games and Economic Behavior, 82:562 - 581, 2013.

[5] H. Aziz, S. Gaspers, S. Mackenzie, and T. Walsh. Fair assignment of indivisible objects under ordinal preferences. In Proc. AAMAS 2014, to appear, May 2014.

[6] M. Babaioff, N. Immorlica, D. Kempe, and R. Kleinberg. Online auctions and generalized secretary problems. SIGecom Exchanges, 7(2), 2008.

[7] S. Bade. Random serial dictatorship: The one and the only. Unpublished manuscript, available from http://www.coll.mpg.de/sites/www.coll.mpg.de/files/ workshop/newproof5.pdf, 2013.

[8] A. Bhalgat, D. Chakrabarty, and S. Khanna. Social welfare in one-sided matching markets without money. In Proc. APPROX+RANDOM '11, volume 6845 of $L N C S$, pages 87-98. Springer, 2011.

[9] A. Bogomolnaia and H. Moulin. A new solution to the random assignment problem. Journal of Economic Theory, 100(2):295 - 328, 2001.

[10] A. Bogomolnaia and H. Moulin. Random matching under dichotomous preferences. Econometrica, 72(1):257-279, 2004.

[11] A. Bogomolnaia and H. Moulin. Size versus fairness in the assignment problem. Unpublished manuscript, available from http://www.gla.ac.uk/media/media_ 295542_en.pdf, 2013.

[12] K. Cechlárová, P. Eirinakis, T. Fleiner, D. Magos, I. Mourtos, and E. Potpinková. Pareto optimality in many-to-many matching problems. Technical report, IM Preprint series A, no. 4/2013, PJ Šafárik University, Faculty of Science, Institute of Mathematics, December 2013.

[13] D. Chakrabarty and C. Swamy. Welfare maximization and truthfulness in mechanism design with ordinal preferences. In Proc. ITCS 2014, pages 105-120. ACM, May 2014.

[14] N. Chen and A. Ghosh. Algorithms for Pareto stable assignment. In V. Conitzer and J. Rothe, editors, Proc. COMSOC '10, pages 343-354. Düsseldorf University Press, 2010 .

[15] N. R. Devanur, K. Jain, and R. Kleinberg. Randomized primal-dual analysis of RANKING for online bipartite matching. In Proc. SODA '13, pages 101-107. ACMSIAM, 2013. 
[16] N.B. Dimitrov and C.G. Plaxton. Competitive weighted matching in transversal matroids. Algorithmica, 62(1-2):333-348, 2012.

[17] S. Dughmi and A. Ghosh. Truthful assignment without money. In Proc. EC '10, pages 325-334. ACM, 2010.

[18] P. Gärdenfors. Assignment problem based on ordinal preferences. Management Science, 20(3):331-340, 1973.

[19] A. Hylland and R. Zeckhauser. The efficient allocation of individuals to positions. Journal of Political Economy, 87(2):293-314, 1979.

[20] P. Jaramillo and V. Manjunath. The difference indifference makes in strategy-proof allocation of objects. Journal of Economic Theory, 147(5):1913-1946, 2012.

[21] P. Krysta, D. Manlove, B. Rastegari, and J. Zhang. Size versus truthfulness in the House Allocation problem. Technical Report 1404.5245, Computing Research Repository, Cornell University Library. Available from http://arxiv.org/abs/ 1404.5245 .

[22] D.F. Manlove. Algorithmics of Matching Under Preferences. World Scientific, 2013.

[23] S. Pápai. Strategyproof assignment by hierarchical exchange. Econometrica, 68(6):1403-1433, 2000.

[24] A.D. Procaccia and M. Tennenholtz. Approximate mechanism design without money. ACM Transactions on Economics and Computation, 1(4), 2013. Article number 18.

[25] M. Pycia and M.U. Ünver. Incentive compatible allocation and exchange of discrete resources. Unpublished manuscript, available from http: / pycia.bol.ucla.edu/ pycia-unver-tcbo.pdf, 2014.

[26] A.E. Roth and A. Postlewaite. Weak versus strong domination in a market with indivisible goods. Journal of Mathematical Economics, 4:131-137, 1977.

[27] A.E. Roth, T. Sonmez, and M.U. Ünver. Pairwise kidney exchange. Journal of Economic Theory, 125(2):151-188, December 2005.

[28] D. Saban and J. Sethuraman. The complexity of computing the random priority allocation matrix. In Proc. WINE '13, volume 8289 of $L N C S$, page 421. Springer, 2013. Full version available at http://www.columbia.edu/ js1353/pubs/ rpcomplexity.pdf.

[29] D. Saban and J. Sethuraman. House allocation with indifferences: A generalization and a unified view. In Proc. EC '13, pages 803-820. ACM, 2013.

[30] L.-G. Svensson. Queue allocation of indivisible goods. Social Choice and Welfare, 11(4):323-330, 1994.

[31] A.C.-C. Yao. Probabilistic computations: Toward a unified measure of complexity (extended abstract). In Proc. FOCS 'r7, pages 222-227. IEEE Computer Society, 1977.

[32] L. Zhou. On a conjecture by Gale about one-sided matching problems. Journal of Economic Theory, 52(1):123-135, 1990. 